\title{
Trends and Pattern of Antibiotic Use in Children in Northern Spain. Lessons From Interpreting Data About Antimicrobial Consumption in Outpatients
}

Laura Calle-Miguel ( $\sim$ lcallemiguel@gmail.com )

Central University Hospital of Asturias

Carlos Pérez Méndez

Universitary Hospital of Cabueñes

Elisa García García

Pediatric Primary Health Care System Gijón

Belén Moreno Pavón

Pediatric Primary Health Care System Gijón

Gonzalo Solís-Sánchez

Central University Hospital of Asturias

\section{Research Article}

Keywords: antibiotic, Northern Spain, antimicrobial, outpatients

Posted Date: July 1st, 2021

DOI: https://doi.org/10.21203/rs.3.rs-555371/v1

License: (c) (1) This work is licensed under a Creative Commons Attribution 4.0 International License.

Read Full License 


\section{Abstract}

Monitoring of antibiotic prescription and consumption behavior is crucial. The Access, Watch and Reserve (AWaRe) classification of antibiotics has been recently introduced in order to measure and improve patterns of antibiotic use. In this study, retrospective data about systemic antibiotic consumption (expressed in defined daily dose per 1000 inhabitants per day [DID]) in pediatric outpatients in a region in Northern Spain (around 100000 children up to 14 years old) from 2005 to 2018 were analysed and compared with antibiotic consumption in general population in Spain. The pattern of use was analysed by the percentage of the current AWaRe categories, the Access-to-Watch index and the amoxicillin index. Data were calculated annually and compared into two periods. Mean antibiotic consumption in pediatric outpatients was 14.0 DID (CI 95\% 13.38-14.62). It remained stable throughout the study and was lower than consumption in general population in Spain, particularly from 2016. Changes in the consumption of the main active principles have led to an improvement in the three metrics of the pattern of use. It is important to have a thorough knowledge of the methodology applied in studies about antibiotic consumption. There is a lack of an optimal standardized metric for pediatric population.

\section{Introduction}

Reducing unnecessary and inappropriate use of antibiotics is a public health priority. Continuous monitoring of antibiotic prescription and consumption behavior is essential to control increasing antimicrobial resistance worldwide [1].

The World Health Organization (WHO) launched the Global Antimicrobial Resistance and Use Surveillance System (GLASS) in 2015, that collects data on antimicrobial consumption of 65 countries [2]. The European Centre for Disease Prevention and Control (ECDC), through the European Surveillance of Antimicrobial Consumption Network (ESAC-Net), has been monitoring antimicrobial consumption in community and hospital sectors in 31 European countries since 1997, describing a high variability between countries. There is a north-to-south and west-to-east increasing gradient in terms of antibiotic consumption and bacterial resistance. Spain is one of the European countries with higher rates of antimicrobial consumption and bacterial resistances [3].

The ECDC collects antimicrobial consumption information according to the Defined Daily Dose (DDD) methodology developed by the WHO Collaborating Centre for Drug Statistics Methodology. In January 2019, this organism changed the DDDs for several antimicrobials [4]. Moreover, the WHO introduced the Access, Watch and Reserve (AWaRe) classification of antibiotics as part of the updated 2017 Model List of Essential Medicines: Access antibiotics are used as first or second-line therapies and should be available at an affordable cost, Watch antibiotics are recommended only for specific indications and Reserve antibiotics include those of last resort. In 2019, the category of Not Recommended antibiotics was added to the framework. This classification was developed to measure and drive improvement in antibiotic stewardship efforts on global, regional and national levels and better understanding of national 
patterns of antibiotic use. There is a WHO global and national level target for Access antibiotics to account for at least $60 \%$ of overall antibiotic consumption by 2023 [5].

Antibiotics are among the most commonly prescribed drugs in pediatrics. Nevertheless, units of measure used in most of these monitoring programs are not accurate to control the consumption in this population as they do not take into account the particularity of children's dosage regarding the weight [ 1 , $6]$.

This study aims to describe the antibiotic consumption and to analyse the pattern of antibiotic use in pediatric outpatients in a region in Northern Spain pointing out difficulties when analysing data of antimicrobial consumption, particularly in children.

\section{Material And Methods}

Retrospective and descriptive study about consumption of antibacterials for systemic use (J01 group of the Anatomical Therapeutic Chemical Classification, ATC) in pediatric outpatients (up to 14 years old) from 2005 to 2018 in Principado de Asturias, a region in Northern Spain with 101767 children protected by the National Health System in 2018. Data were collected from the billing data of the containers dispensed in the pharmacy offices run by the National Health System.

Information about antibiotic consumption was expressed as DDD per 1000 inhabitants per day (DID) according to the latest and the previous available ATC/DDD index [3, 7]. DIDs were calculated annually and aggregated by $\mathrm{J} 01$ group, therapeutic group and active principle levels. Global data were contrasted to information about antibiotic consumption in Spanish general population by the ECDC $[3,7]$.

To analyse the pattern of use, antibiotics were classified into the four current AWaRe categories: Access, Watch, Reserve and Not Recommended. Antibiotics that could not be assigned to the AWaRe categories were left unclassified categories [5]. Three metrics of pattern of antibiotic use were calculated. First, the percentage of Access, Watch, Reserve and Not Recommended antibiotic consumption (the DIDs of antibiotics in each group divided by the total DIDs). Second, the Access-to-Watch index (the ratio of DIDs of Access antibiotics to Watch antibiotics). Third, the amoxicillin index (the DIDs of amoxicillin divided by the total DIDs), being the recommended first-line therapy for respiratory tract infections, which are the most common indication for antibiotic prescriptions in pediatrics [8].

Antibiotic consumption and the pattern of use were calculated annually and compared into two periods (2005-2011 and 2012-2018). Descriptive analyses were performed using means and confidence intervals of $95 \%(\mathrm{Cl} 95 \%)$ and variables were compared using the T-Student test. Statistical analyses were performed using IBM SPSS Statistics software (Statistical Package for the Social Sciences) version 23.0. The statistical significance level was defined as a two-tailed $p$-value $<0.05$.

We conducted this study in accordance with the Declaration of Helsinki and its subsequent amendments. All data used were population data. No personal or identifiable information was collected, so it was not 
necessary to request informed consent. Ethics approval was obtained from the Ethics Committee (Comité de Ética de Investigación del Gobierno del Principado de Asturias).

\section{Results}

Mean antibiotic consumption in pediatric outpatients in Asturias (2005-2018) was 14.0 DID (CI 95\% 13.38-14.62), showing no statistically significant changes between periods. Figure 1 represents evolution of consumption of antibacterials for systemic use in the pediatric population in the community sector, compared to consumption in Spanish general population published by the ECDC, according to the previous and the latest ATC/DDD index [3, 7].

The most consumed therapeutic groups were J01C ( $\beta$-lactam penicillins, 10.7 DID; 76.7\%), J01F (macrolides, lincosamides and streptogramins, $1.8 \mathrm{DID} ; 12.8 \%$ ) and J01D (other $\beta$-lactam antibacterials, referring to cephalosporins, $1.2 \mathrm{DID} ; 8.9 \%$ ). The consumption of ten active principles represented $97.6 \%$ of the global use and the consumption of amoxicillin and amoxicillin-clavulanate accounted for $72.8 \%$. Consumption of amoxicillin, phenoxymethylpenicillin, azithromycin and doxycycline significantly increased, whereas consumption of cefuroxime, cefixime and clarithromycin decreased throughout the study (Table 1). 
Table 1

Consumption (DID) of total and most commonly used antibiotics and pattern of use in pediatric outpatients in Asturias. Global data and comparison between periods.

\begin{tabular}{|c|c|c|c|c|c|}
\hline & Code & Global & $\begin{array}{l}\text { Period } \\
2005- \\
20011\end{array}$ & $\begin{array}{l}\text { Period } \\
\text { 2012-2018 }\end{array}$ & $p$ \\
\hline \multicolumn{6}{|l|}{ Global consumption } \\
\hline $\begin{array}{l}\text { Antibacterials for systemic } \\
\text { use }\end{array}$ & J01 & $\begin{array}{l}14(13.38- \\
14.62)\end{array}$ & $\begin{array}{l}13.99 \\
(13.04- \\
14.95)\end{array}$ & $\begin{array}{l}14(12.91- \\
15.1)^{-}\end{array}$ & 0.949 \\
\hline \multicolumn{6}{|c|}{ Most commonly consumed antibiotics } \\
\hline $\begin{array}{l}\text { Amoxicillin } \\
\text { (Access) }\end{array}$ & J01CA04 & $\begin{array}{l}5.16(4.79- \\
5.52)\end{array}$ & $\begin{array}{l}4.76(4.22- \\
5.3)\end{array}$ & $\begin{array}{l}5.55(5.18- \\
5.93)\end{array}$ & 0.013 \\
\hline $\begin{array}{l}\text { Amoxicillin-clavulanate } \\
\text { (Accesss) }\end{array}$ & J01CR02 & $\begin{array}{l}5.03(4.74- \\
5.32)\end{array}$ & $\begin{array}{l}5.23(4.9- \\
5.56)\end{array}$ & $\begin{array}{l}4.84(4.30- \\
5.38)\end{array}$ & 0.180 \\
\hline $\begin{array}{l}\text { Azithromycin } \\
\text { (Watch) }\end{array}$ & J01FA10 & $\begin{array}{l}1.04(0.86- \\
1.21)\end{array}$ & $\begin{array}{l}0.79(0.67- \\
0.91)\end{array}$ & $\begin{array}{l}1.28(1.11- \\
1.46)\end{array}$ & 0.002 \\
\hline $\begin{array}{l}\text { Cefuroxime } \\
\text { (Watch) }\end{array}$ & J01DC02 & $\begin{array}{l}0.82(0.69- \\
0.96)\end{array}$ & $\begin{array}{l}1.00(0.93- \\
1.06)\end{array}$ & $\begin{array}{l}0.65(0.46- \\
0.84)\end{array}$ & 0.004 \\
\hline $\begin{array}{l}\text { Clarithromycin } \\
\text { (Watch) }\end{array}$ & J01FA09 & $\begin{array}{l}0.61(0.48- \\
0.73)\end{array}$ & $\begin{array}{l}0.80(0.76- \\
0.84)\end{array}$ & $\begin{array}{l}0.42(0.30- \\
0.54)\end{array}$ & 0.002 \\
\hline $\begin{array}{l}\text { Phenoxymethylpenicillin } \\
\text { (Access) }\end{array}$ & J01CE02 & $\begin{array}{l}0.33(0.29- \\
0.36)\end{array}$ & $\begin{array}{l}0.29(0.26- \\
0.32)\end{array}$ & $\begin{array}{l}0.37(0.32- \\
0.41)\end{array}$ & 0.009 \\
\hline $\begin{array}{l}\text { Cefixime } \\
\text { (Watch) }\end{array}$ & J01DD08 & $\begin{array}{l}0.32(0.26- \\
0.38)\end{array}$ & $\begin{array}{l}0.39(0.35- \\
0.43)\end{array}$ & $\begin{array}{l}0.25(0.16- \\
0.34)\end{array}$ & 0.009 \\
\hline $\begin{array}{l}\text { Benzathine } \\
\text { phenoxymethylpenicillin } \\
\text { (Unclassified) }\end{array}$ & J01CE10 & $\begin{array}{l}0.21(0.19- \\
0.24)\end{array}$ & $\begin{array}{l}0.2(0.16- \\
0.24)\end{array}$ & $\begin{array}{l}0.23(0.19- \\
0.26)\end{array}$ & 0.277 \\
\hline $\begin{array}{l}\text { Doxycycline } \\
\text { (Access) }\end{array}$ & J01AA02 & $\begin{array}{l}0.09(0.06- \\
0.11)\end{array}$ & $\begin{array}{l}0.05(0.04- \\
0.06)\end{array}$ & $\begin{array}{l}0.12(0.1- \\
0.15)\end{array}$ & 0.002 \\
\hline
\end{tabular}

Data presented correspond to means and confidence interval of $95 \%$. Numbers in bold font: statistically significant differences $(p<0.05)$.

DID: Defined Daily Dose (DDD) per 1000 inhabitants per day. 


\begin{tabular}{|c|c|c|c|c|c|}
\hline & Code & Global & Period & Period & $p$ \\
\hline & & & $\begin{array}{l}2005- \\
20011\end{array}$ & 2012-2018 & \\
\hline $\begin{array}{l}\text { Trimethoprim/ } \\
\text { Sulfamethoxazole (Access) }\end{array}$ & J01EE01 & $\begin{array}{l}0.06(0.05- \\
0.06)\end{array}$ & $\begin{array}{l}0.06(0.05- \\
0.07)\end{array}$ & $\begin{array}{l}0.06(0.05- \\
0.06)\end{array}$ & 0.949 \\
\hline Pattern of antibiotic use & & & & & \\
\hline Access antibiotics (\%) & & $\begin{array}{l}76.47 \% \\
(75.02- \\
77.92)\end{array}$ & $\begin{array}{l}74.46 \\
(73.11- \\
75.81)\end{array}$ & $\begin{array}{l}78.48 \\
(77.12- \\
79.84)\end{array}$ & 0.002 \\
\hline Access-to-Watch index & & $\begin{array}{l}3.58(3.29- \\
3.88)\end{array}$ & $\begin{array}{l}3.18(2.95- \\
3.4)\end{array}$ & $\begin{array}{l}3.99(3.67- \\
4.30)\end{array}$ & 0.002 \\
\hline Amoxicillin index & & $\begin{array}{l}0.37(0.35- \\
0.39)\end{array}$ & $\begin{array}{l}0.34(0.32- \\
0.36)\end{array}$ & $\begin{array}{l}0.4(0.38- \\
0.41)\end{array}$ & 0.002 \\
\hline $\begin{array}{l}\text { Data presented correspond } t \\
\text { statistically significant diffel }\end{array}$ & $\begin{array}{l}\text { ans and } \\
S(p<0.0\end{array}$ & Idence inter & $95 \%$. Num & in bold font: & \\
\hline DID: Defined Daily Dose (DD & $1000 \mathrm{ir}$ & itants per da & & & \\
\hline
\end{tabular}

The consumption of Access antibiotics was greater than $70 \%$ every year (minimum of $71.5 \%$ in 2005 , maximum of $80.4 \%$ in 2015; Fig. 2) and both Access-to-Watch index and amoxicillin index significantly increased overtime (Table 1).

\section{Discussion}

The present study, carried out in the entire pediatric population in a region in Northern Spain during 14 years, has shown that antibiotic consumption in the pediatric community sector in our region was lower than consumption in general population in Spain, particularly from 2016, and that the pattern of antibiotic use improved throughout the study.

Nevertheless, readers must be aware of several aspects about interpreting data of antimicrobial consumption. First, the ECDC collects antimicrobial consumption information according to the DDD methodology developed by the WHO Collaborating Centre for Drug Statistics Methodology. One DDD is the assumed average maintenance dose per day for a drug used in its main indication in a $70 \mathrm{~kg}$ adult. DDD is a technical unit of measurement, not a standard for appropriate use, but it makes possible to aggregate information about substances with different pack sizes, strengthening it into units of measurement of active substances. DID represents a standard in performing valid and reliable crossnational or longitudinal studies of antimicrobial consumption and, thus, it allows to compare data between regions and countries. DID has been selected as the primary harmonised outcome indicator by the ECDC, the European Food Safety Authority (EFSA) and the European Medicines Agency (EMA) $[9,10]$. 
DDD values may be modified over time because of changes in the main indication or amendments for the recommended or prescribed daily dose. In January 2019, the WHO Collaborating Centre for Drug Statistics Methodology changed the DDDs for several antimicrobials, including those with the highest consumption in the Spanish community sector: amoxicillin and amoxicillin-clavulanate (both from $1 \mathrm{~g}$ to 1.5g) [4]. Historical ESAC-Net data on antimicrobial consumption since 1997 were recalculated accordingly, following the normative to report all data from all years with the latest available ATC/DDD index. Thus, recent data displayed in the interactive database differ from those in historical annual epidemiological reports. Data may appear to be lower at present than previously, with the largest impact for the penicillins group $[3,4,7,9]$. Readers must be aware of these changes when comparing current data with that published in scientific papers until 2019. We observed a decline around $25 \%$ of antimicrobial consumption rates in our pediatric population according to the latest ATC/DDD index, similar to the difference observed in Spanish general population after the ATC/DDD update [7, 9-10].

Second, there has been a noticeable increase of antimicrobial consumption rates in Spain since 2016. Spain changed the source to report community consumption data from 2016 onwards, from reimbursement data to National Health System to sales data, which resulted in a substantial technical increase of antimicrobial consumption compared with previous years. The major limitation of reimbursement data is that antimicrobials dispensed without a prescription and non-reimbursed prescribed antimicrobials are not included. Spanish data are currently more reliable and comparable with that of other countries, as just a quarter of countries reporting information to ESAC-Net still provide reimbursement data [3]. Rates of Spanish antimicrobial consumption are worrisome. In 2019, Spain was the fifth European country with the highest antimicrobial consumption in the community sector, reaching 23.3 DID and above the European mean (18 DID). A significant decrease in the mean consumption of antibacterials in Europe was observed over the last ten years [9]. There is a high prescription of antimicrobials in the private sector in several regions in Spain [11]. We do not expect to have a high rate of non-reimbursed prescribed antimicrobials in our population, as $98 \%$ of the pediatric population is covered by the National Health System.

Third, prescriptions in children are commonly based on patient weight. Measuring antimicrobial consumption by DDD can be found questionable in children as it quantifies the volume of antimicrobial administered but does not take into account body weight. Over other 25 different measures can be found to describe antimicrobial use in pediatric population, referring to the proportion of exposed patients, quantity and duration of antimicrobial therapy [12]. Current recommendation metric in hospitalized patients for pediatric antimicrobial stewardship programs is the number of Days of Therapy (DOT). Antimicrobial DDD adjusted by weight has been recently developed for pediatric inpatients; this measure is highly correlated with DOT and names DDD in $\mathrm{mg} / \mathrm{kg}$, a standard not defined by the ATC/DDD system $[1,6]$. These measures have their application in the hospital sector and require patient-level data, often inaccesible in studies in the community sector. DDD can still be used to follow antimicrobial use density in populations where patients' average weight is constant, as it could be in an entire pediatric population in a region as ours $[12,13]$. 
In relation to the pattern of antibiotic use, the majority of non-Access antibiotic consumption in our study was from Watch Antibiotics, with Reserve and Not Recommended antibiotic consumption accounting for less than $0.15 \%$. We observed an improvement in the pattern of antibiotic use in our population in three mentioned metrics. Azithromycin was the main Watch antibiotic whose consumption increased over time in our study and the active principle with the highest increase in our region [13]. Spain achieved the target in the WHO AWaRe framework of at least $60 \%$ of Access antibiotics in general population in 2004 and has increased since then up to $68.5 \%$ in 2015 [14]. Access antibiotics in our population were above $70 \%$ every year. However, our data are still inferior to that presented in the pediatric Spanish population, notifying $90 \%$ of Access antibiotics, Access-to-Watch index beyond 10 and amoxicillin index around 0.5 in 2015 [8].

The main current and global problem is the increasing consumption of both Access and Watch antibiotics, with the consumption of Watch antibiotics rising much faster than Access antibiotics, especially in low and middle-income countries. This challenges to achieve the AWaRe target for Access antibiotics to account for $60 \%$ of the global consumption by 2023 in these countries. The use of the amoxicillin index can inform the development of initiatives to improve the use of amoxicillin and other Access antibiotics. Countries characterized by high consumption of amoxicillin have national guidelines for the treatment of respiratory tract infections supporting the use of this drug. The AWaRe antibiotic classification provides a useful framework for exploring national antibiotic consumption patterns $[5,8$, 14].

Our study presents several limitations, mostly derived from the analysis and comparison of antibiotic consumption giving the already mentioned particularities of the different populations and measures and changes on the methodology. We did not include data about the hospital sector, below $1.5 \%$ of global consumption in our population, being the objective to analyse the consumption and the pattern of antibiotic use in the pediatric community. We did not include data about antibiotic consumption without prescription, being this sale prohibited in Spain since 2002, or about prescription of antimicrobials in the private sector, not expecting, as already mentioned, to have a high rate of non-reimbursed prescribed antimicrobials in our population.

In conclusion, antibiotic consumption in pediatric outpatients in Northern Spain along 2005-2018 period remained stable and was lower than consumption in general population in Spain. The pattern of antibiotic use improved throughout the study, but there are still some points of improvement as increasing the amoxicillin index or controlling consumption of some Watch antibiotics such as azithromycin. There is still a lack of an optimal standardized metric for pediatric outpatients. Different methods of measurements and major changes on the data collection methodology used by the main international consumption surveillance systems must be taken into account when interpreting and comparing data about antibiotic consumption.

\section{Declarations}


Acknowledgements. Authors express their gratitude to Gracia Modroño Riaño and Maria Isabel Iglesias Carbajo (specialists in Hospital Pharmacy and collaborators of this study) and to Ernesto Sánchez Villares Foundation (from the Sociedad de Pediatría de Asturias, Cantabria y Castilla y León, SCCALP) for the donation of a grant ( $€ 500$ ) to LCM for the development of this study.

Authors' contributions. All authors contributed to the study conception and design. Conceptualization was performed by CPM. Material preparation and data collection were performed by LCM and GSS. Analysis was performed by GSS, EGG and LCM. The first draft of the manuscript was written by LCM and BMP. All authors commented on previous versions of the manuscript, read, and approved the final manuscript. Authors have not had any prior discussions with a Scientific Reports Editorial Board Member about the work described.

Conflicts of interest: LCM has received a grant ( $€ 500)$ from Ernesto Sánchez Villares Foundation (from the Sociedad de Pediatría de Asturias, Cantabria y Castilla y León, SCCALP) for the development of this study. The remain authors have no conflicts of interest to disclose.

Data statement: To collect the data, a permission from the Sub-Direction of the Organization of Health System of Principado de Asturias was obtained. All the authors have full access to the data and LCM is the guarantor for the data.

Ethics approval: No personal or identifiable data were collected. Ethics approval was obtained from the Comité de Ética de Investigación del Gobierno del Principado de Asturias.

\section{References}

1. Ibrahim, O. M. \& Polk, R. E. Antimicrobial use metrics and benchmarking to improve stewardship outcomes. Methodology, opportunities and challenges. Infect Dis Clin N Am, 28, 195-214 (2014).

2. World Health Organization. Global antimicrobial resistance surveillance system (GLASS) report: early implementation 2020. Geneva, Switzerland. ISBN 978-92-4-000558-7. (2020)

3. European Center for Disease Prevention and Control. Antimicrobial consumption database (ESACNet) [ESAC-Net web site]. https://www.ecdc.europa.eu/en/antimicrobial-consumption/surveillanceand-disease-data/database (2020).

4. World Health Organization. ATC/DDD [ATC/DDD website]. Norway: WHO Collaborating Centre for Drug Statistics Methodology. https://www.whocc.no/atc_ddd_methodology/purpose_of_the_atc_ddd_system (2020).

5. WHO. World Health Organization model list of essential medicines, 21st list, 2019 (World Health Organization, Geneve, 2019).

6. Montecatine-Alonso, E. et al. Antimicrobial defined daily dose adjusted by weight: a proposal for antibiotic consumption measurement in children. Enferm Infecc Microbiol Clin, 37 (5), 301-306 (2019). 
7. European Centre for Disease Prevention and Control. Antimicrobial consumption in the EU/EEA, annual epidemiological report for 2017 (ECDC, Stockholm, 2018).

8. Hsia, Y. et al. Consumption of oral antibiotic formulations for young children according to the WHO Access, Watch, Reserve (AWaRe) antibiotic groups: an analysis of sales data from 70 middle-income and high-income countries. Lancet Infect Dis, 19 (1), 67-75 (2019).

9. European Centre for Disease Prevention and Control. Antimicrobial consumption in the EU/EEA, annual epidemiological report for 2019 (ECDC, Stockholm, 2020).

10. European Centre for Disease Prevention and Control. Antimicrobial consumption (AMC) reporting protocol 2020. European Surveillance of Antimicrobial Consumption Network (ESAC-Net) surveillance data for 2019 (ECDC, Stockholm, 2020).

11. de Sanidad, M. Servicios Sociales e Igualdad. Plan Nacional de Resistencia Antibióticos [PRAN web site]. http://www.resistenciaantibioticos.es/es (2020).

12. Fortin, E. et al. C. Measuring antimicrobial use in hospitalized patients: a systematic review of available measures applicable to paediatrics. J Antimicrob Chemother, 69 (6), 1447-1456 (2014).

13. Calle-Miguel, L. et al. Evolution of antibiotic consumption in pediatric outpatients of Asturias, Spain (2005-2018). An Pediatr 23: S1695-4033(20)30496-3(2021).

14. Klein, E. Y. et al. Assessment of WHO antibiotic consumption and access targets in 76 countries, 2000-15: an analysis of pharmaceutical sales data. Lancet Infect Dis, 21 (1), 107-115 (2020).

\section{Figures}




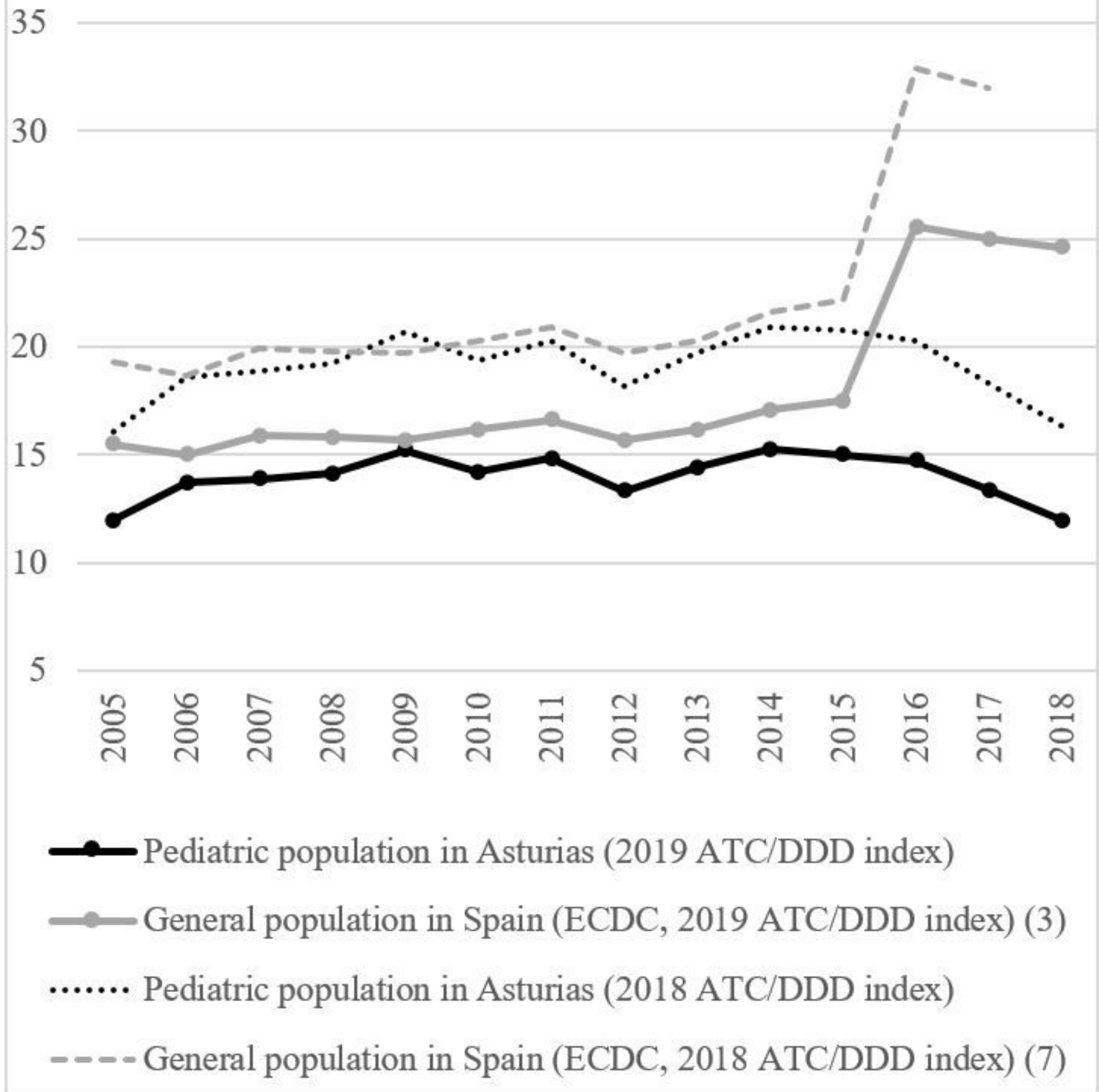

\section{Figure 1}

Antibiotic consumption (DID) in the community sector in pediatric outpatients in Asturias and Spanish general population (2005-2018). DID: Defined Daily Dose (DDD) per 1000 inhabitants per day. 


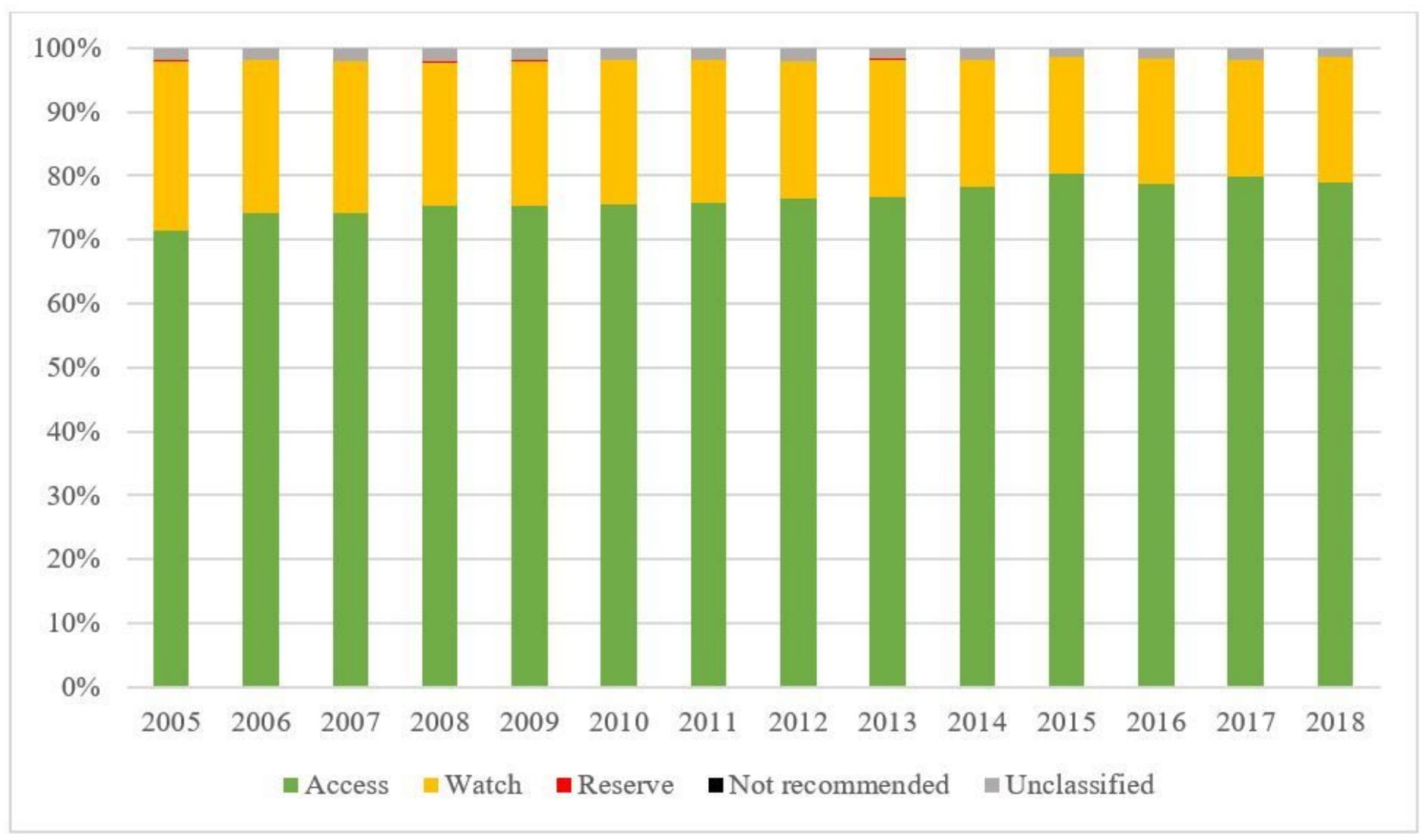

Figure 2

Consumption of antibiotics (\%) according to the AWaRe classification in pediatric outpatients in Asturias (2005-2018). AWaRe: Access, Watch and Reserve antibiotics 